\title{
AGROMETEOROLOGIA
}

\section{QUANTIFICAÇÃO DA SECA AGRÍCOLA PELO ÍNDICE PADRONIZADO DE EVAPOTRANSPIRAÇÃO REAL (IPER) NO ESTADO DE SÃO PAULO $\left(^{(1)}\right.$}

\author{
GABRIEL CONSTANTINO BLAIN $\left(\left(^{*}\right)\right.$; ORIVALDO BRUNINI $\left.{ }^{2,3}\right)$
}

\begin{abstract}
RESUMO
Apesar do seu grande desenvolvimento tecnológico atual, a agricultura paulista é freqüentemente castigada por adversidades climáticas; entre essas, a seca agrícola é a de efeito mais duradouro e de maior intensidade. Com base em séries históricas de evapotranspiração real (ETR), ajustadas à distribuição beta, o objetivo desse trabalho foi adaptar a metodologia do Índice Padronizado de Precipitação (SPI) aos interesses agrícolas. As análises permitiram concluir que tal ajuste resultou em novo índice, Índice Padronizado de Evapotranspiração Real (IPER), consistente para a padronização e monitoramento "próximo ao tempo real" da seca agrícola na escala decendial no Estado de São Paulo. Notou-se ainda que a variação desse índice está relacionada ao número de desvios-padrão que um dado valor de ETR está abaixo do valor climaticamente esperado para um dado período e local, indicando dessa maneira a intensidade/ severidade de uma seca agrícola padronizada para cada local.
\end{abstract}

Palavras Chaves: Índice Padronizado de Precipitação, monitoramento próximo ao tempo real.

\section{ABSTRACT \\ AGRICULTURAL DROUGHT QUANTIFICATION USING THE ACTUAL EVAPOTRANSPIRATION INDEX (IPER) IN STATE OF SÃO PAULO, BRAZIL}

Agricultural drought has always brought serious problems related to food production in the State of São Paulo, Brazil. The scope of this study was to adapt the methodology of the Standardized Precipitation Index (SPI) to the historical values of Actual Evapotranspiration series fitted to the beta distribution, to quantify agricultural drought in the State of São Paulo. The new index is named Standardized Actual Evapotranspiration Index (IPER). The results indicates that such new index, IPER, is vey useful to the "near real time monitoring" of the agricultural drought in a ten day period. It was observed that the range of this new index is directly related to the numbers of standard deviation for which a given value of actual evapotranspiration is below of its expected value, indicating the intensity or severity of the agricultural drought.

Key Words:Standardized Precipitation Index, near real time monitoring.

$\left({ }^{1}\right)$ Recebido para publicação em 22 de junho de 2005 e aceito em 16 de maio de 2006.

$\left({ }^{2}\right)$ Centro de Pesquisa e Desenvolvimento de Ecofisiologia e Biofísica, Instituto Agronômicos (IAC), Caixa Postal 28, 13020-902 Campinas (SP). E-mail: brunini@iac.sp.gov.br; gabriel@iac.sp.gov.br. *Autor correspondente.

$\left({ }^{3}\right)$ Com Bolsa de Produtividade em Pesquisa do CNPq. 


\section{INTRODUÇÃO}

A seca é um fenômeno meteorológico complexo, com influências sociais freqüentemente agravadas pela ação humana. A ocorrência dessa anomalia climática, em várias regiões do globo terrestre, na década de setenta evidenciou a vulnerabilidade (4) do homem a esse risco climático, demonstrando a necessidade de melhor entendimento, melhor previsão de sua ocorrência e uso correto de medidas mitigatórias (WILHITE e GLANTZ,1987).

Segundo Heim Junior (2002), o grande número de setores afetados pela seca, sua diversividade geográfica, a distribuição temporal e a demanda provocada pela ação humana tornam difícil o desenvolvimento de uma definição universal desse evento. Tal confusão na definição desse fenômeno natural pode resultar em falta de entendimento das reais implicações sociais de uma seca, tornando ineficazes as medidas de combate a essa anomalia climática (Wilhite e GLANTZ,1987).

A maior parte dos trabalhos científicos reconhece quatro tipos de seca: meteorológica, hidrológica, agrícola e socioeconômica (WiLhite e GlantZ,1987 e RASMUSSEN et al., 1993).

Keyantash e Dracup (2004) afirmam que, a menos que a demanda por água da sociedade exceda significativamente o suprimento natural, a seca socioeconômica é uma conseqüência dos demais tipos de seca, sendo caracterizada monetariamente.

A seca meteorológica é o resultado de um déficit de precipitação pluvial; a hidrológica é uma deficiência no volume de água disponível, incluindo lençol freático, reservatórios e rios (WILHITE, 2000). O primeiro tipo de seca pode se desenvolver rapidamente e "terminar abruptamente" enquanto a hidrológica necessita de um período prolongado de déficit de precipitação pluvial, persistindo por mais tempo que a meteorológica (Heim JunIor, 2002).

$\left.{ }^{4}\right)$ Grau que um sistema é susceptível, ou incapaz de enfrentar efeitos adversos. É uma função entre a natureza, a magnitude e o percentual de uma variação que o sistema é exposto, sua sensitividade, sua capacidade adaptativa. Depende, ainda, de uma relação complexa de situações econômicas, sociais, culturais e políticas, que são configuradas por eventos extremos já enfrentados por determinadas sociedades (IPCC, 2001)
A seca agrícola está relacionada à baixa disponibilidade de umidade no solo a qual torna o suprimento de água às culturas insuficiente para repor as perdas por evapotranspiração (WORLD Meteorological Organization, 1975). Além de estar relacionada a fases críticas do desenvolvimento/ crescimento dos vegetais, o início de uma seca agrícola pode se distanciar do de uma meteorológica, pois depende quase que exclusivamente da água disponível na zona radicular das culturas no solo.

O fechamento dos estômatos das plantas, causado por uma deficiência hídrica na zona radicular, além de tornar o processo fotossintético limitado, relaciona diretamente esse efeito negativo sobre o desenvolvimento vegetal. Tal premissa deu suporte a PALMER (1968) quando afirmou que, de forma simplista, uma seca agrícola nada mais é que um déficit de evapotranspiração. Entretanto, segundo esse autor, tal deficiência, não deve ser calculada com base na evapotranspiração potencial (ETP), já que em estações climatologicamente secas, um valor de evapotranspiração real (ETR) inferior ao da evapotranspiração potencial não significa, necessariamente, a existência de uma anomalia climática. Uma seca agrícola está, portanto, relacionada ao déficit entre o valor da evapotranspiração real, estimada em um período, e o valor climatologicamente esperado para o mesmo período em questão. $\mathrm{O}$ estudo da seca agrícola exige ainda uma escala de tempo "pequena" (menor do que um mês) para que seu efeito seja corretamente avaliado (PAlmer, 1968; HAYes et al., 1999).

A implementação de sistemas de monitoramento em "tempo real" e "estações de aviso" são, segundo Wilhite (1982), a única forma dos "estudiosos da atmosfera" contribuírem com as ações de combate à seca nos Estados Unidos da América do Norte. Tais contribuições poderiam ser feitas por meio de estudos realizados sobre índices utilizados para quantificar as condições hídricas das áreas em questão. No caso de tais ferramentas proverem informações inadequadas ou imprecisas, devem ser re-avaliados ou até mesmo substituídas por novos índices.

Uma das ferramentas mais adequadas para a quantificação da seca, sob o ponto de vista meteorológico, é o Índice Padronizado de Precipitação (SPI). Desenvolvido por Mckee et al. (1993), tal quantificador vem sendo utilizado em diversos países do mundo, nos programas e ações governamentais de combate e mitigação à seca. Tal índice é capaz de monitorar essa anomalia climática em diversas escalas de tempo, reconhecendo, portanto, diferentes tipos de seca em sua análise. Entretanto, o fato de utilizar apenas dados de precipitação pluvial distancia o uso 
desse quantificador dos interesses agrícolas. O SPI tem como base de sua metodologia o ajuste de séries de precipitação pluvial à distribuição gama incompleta. Tal distribuição é adequada para descrever o comportamento de variáveis que possuam limite inferior ou superior a 0 .

BRUNINI et al. (2002) afirmam que os enormes prejuízos sofridos pela agricultura paulista, em 1963 e 1964, foram causados por uma seca extrema ocorrida nesse Estado. Esses autores afirmam ainda, que apesar do seu grande desenvolvimento tecnológico atual, a agricultura paulista é freqüentemente castigada por adversidades climáticas, sendo a seca a que tem efeito mais duradouro e de maior intensidade.

Recomenda-se o uso da distribuição beta para a caracterização da variabilidade temporal das séries de ETR no Estado de São Paulo e conseqüentemente seu uso no desenvolvimento de um índice de seca agrícola padronizado no tempo e no espaço BLAIN e BRUNINI $\left({ }^{4}\right)$. Os mesmos autores ressaltam ainda que as características da distribuição beta, como seu intervalo de trabalho $(0,1)$, devem ser observadas no caso de um índice destinado ao monitoramento "próximo ao tempo real" da seca agrícola em uma região.

Assim, esse estudo tem como objetivo a adaptação da metodologia do SPI para a quantificação da seca agrícola, em escala decendial, com base no ajuste de séries de evapotranspiração real, estimadas pelo método do balanço hídrico de THORnthwaite e MATHER (1955), à distribuição beta.

\section{MATERIAL E MÉTODOS}

Com base em dados da rede de Estações Meteorológicas do Instituto Agronômico da Secretaria de Agricultura e Abastecimento, foram gerados balanços hídricos decendais com período homogêneo, entre 1971 e 2000, para as localidades de Campinas (22 $\left.54^{\circ} \mathrm{S} ; 47^{\circ} 05^{\prime} \mathrm{W} ; 669 \mathrm{~m}\right)$, Jaú $\left(23^{\circ} 17^{\prime} \mathrm{S} ; 48^{\circ} 38^{\prime} \mathrm{W} ; 580\right.$ $\mathrm{m})$, Ribeirão Preto $\left(21^{\circ} 11^{\prime} \mathrm{S} ; 47^{\circ} 48^{\prime} \mathrm{W} ; 620 \mathrm{~m}\right)$, Mococa $\left(21^{\circ} 28^{\prime} \mathrm{S} ; 51^{\circ} 05^{\prime} \mathrm{W} ; 665 \mathrm{~m}\right)$, Pindorama $\left(21^{\circ} 13^{\prime} \mathrm{S}\right.$ $\left.48^{\circ} 56^{\prime} \mathrm{W} ; 562 \mathrm{~m}\right)$ e Ubatuba $\left(23^{\circ} 27^{\prime} \mathrm{S} 45^{\circ} 04^{\prime} \mathrm{W} ; 8 \mathrm{~m}\right)$.

$\left({ }^{4}\right)$ BLAIN, G. C. BRUNINI, O. Caracterização do regime de evapotanspiração real, em escala decendial, no Estado de São Paulo. Revista Brasileira de Meteorologia, Santa Maria. (Submetido à publicação)
Essas regiões representam os tipos climáticos, de acordo com a classificação de Köeppen, espacialmente preponderantes no estado, sendo eles: Cwa (Campinas, Jaú e Mococa), AW (Ribeirão Preto e Pindorama) e Af (Ubatuba). A escolha desses postos também considerou a disponibilidade de série homogênea com data inicial em janeiro de 1971 ou anterior.

Os valores de ETR gerados em tais balanços, foram agrupados em decêndios, resultando em 36 séries para cada local. Blain e Brunini (dados não publicados) comprovam a aderência desse elemento, em escala decendial, à função densidade de probabilidade (problably density function, PDF) beta. Após essa avaliação, a probabilidade cumulativa de um dado valor estimado de ETR é calculada. A função normal inversa (Gaussiana), que possui média zero e variância unitária é aplicada à probabilidade acumulada. $\mathrm{O}$ resultado é o valor do novo índice, denominado "Índice Padronizado de Evapotranspiração Real" (IPER). O método é descrito a seguir:

A distribuição beta é definida no intervalo $(0,1)$ com a seguinte função densidade de probabilidade da variável $x$.

$$
f(X)=\frac{X^{(\alpha-1)-(1-X)^{\beta-1}}}{\beta(\alpha, \beta)}
$$$$
\text { para } 0<x>1
$$

Os parâmetros $\alpha$ e $\beta$ determinam a forma da distribuição e são calculados da seguinte forma:

$$
\begin{aligned}
& \beta=\left[\frac{\overline{\mathrm{x}}(1-\overline{\mathrm{x}})}{\mathrm{S}^{2}}-1\right] *(1-\bar{x}) \\
& \alpha=\frac{\bar{x}^{2}(1-\bar{x})}{S^{2}}
\end{aligned}
$$

Em que:

$\bar{x}$ a média aritmética dos valores de $\mathrm{x}$ na série considerada;

$s^{2} a$ variância da série de valores de $x$ considerada.

Segundo Blain e Brunini (dados não publicados), foi utilizada a seguinte transformação de variáveis, para que a distribuição beta pudesse ser aplicada às séries decendiais de ETR.

$$
\text { ETR' }^{\prime}(\text { ETR- } a) /(b-a)
$$

Em que:

ETR '- Variável evapotranspiração real transformada de tal modo que $0<$ ETR $^{\prime}<1$;

a - Menor valor da série de dados; e

b - Maior valor da série de dados. 
Entretanto, esses autores ainda ressaltam que, em uma longa série de dados ajustados à distribuição beta, magnitudes de ETR decendial abaixo do menor valor da série (a) e acima do maior valor desta (b), indicam probabilidade de ocorrência indefinida. Entretanto, no monitoramento contínuo de uma região (função comumente atribuída à índices de seca), casos extremos de ocorrência de magnitudes de ETR abaixo do menor valor da série histórica utilizada para a caracterização da distribuição beta, podem ser detectados. Tal fato poderá resultar na indefinição do valor final de um índice de seca.

Considerando-se a afirmação acima citada e o fato de que as temperaturas médias diárias, no Estado de São Paulo, impossibilitam valores de ETR decendiais acima de $100 \mathrm{~mm}$, optou-se pela seguinte transformação de variável:

$$
\mathrm{ETR}^{\prime \prime}=\mathrm{ETR} / 100
$$

Em que:

ETR" Variável evapotranspiração real transformada de tal modo que $0<$ ETR" $<1$; G(ETR") é então transformada em uma variável normal (valor final do IPER) por meio das equações desenvolvidas por AbRAMOWITZ e STEGUN (1965).

$$
\begin{aligned}
& \text { IPER }=-\left(t-\frac{c o+c_{1} t+c_{2} t^{2}}{1+d_{1} t+d_{2} t^{2}+d_{3} t^{3}}\right) \text { para } 0<\mathrm{H}(\mathrm{x}) \leq 0,5 \\
& \text { IPER }=+\left(t-\frac{c o+c_{1} t+c_{2} t^{2}}{1+d_{1} t+d_{2} t^{2}+d_{3} t^{3}}\right) \text { para } 0,5<\mathrm{H}(\mathrm{x})<0,5
\end{aligned}
$$

Em que:

$$
\begin{aligned}
& t=\sqrt{\left(\ln \left(\frac{1}{(G(E T R))^{2}}\right)\right.} \text { para } 0<0,5 \\
& t=\sqrt{\left(\ln \left(\frac{1}{(1-G(E T R))^{2}}\right)\right.} \text { para } 0,5<<1
\end{aligned}
$$

Em que:

$$
c_{c o}=2,515517 ;{ }_{c 1}=0,802853 ; c_{c 2}=0,010328 ;{ }_{d 1}=
$$

$$
1,432788 ;_{d 2}=0,189269 ; d 3=0,001308
$$

Nos valores do IPER próximos ou superiores a 0, nota-se que a ETR acumulada em um dado decêndio está próxima ou superior ao valor climatologicamente esperado desse parâmetro nesse período. Em valores negativos do índice, observa-se que a evapotranspiração real de um dado decêndio está abaixo do patamar esperado para o período em questão.

Foram ainda gerados balanços hídricos decendiais para todas as localidades em questão para 2004 a 2005. A variação da deficiência hídrica, estimada nesse período de acordo com método de
THornthwaite e Mather (1955), e a variação do IPER foram comparadas em todos os locais do estudo.

Assim como descrito em Blain (2005), o fenômeno seca é entendido, nesse estudo, como uma anomalia climática. Com isso, pode-se assumir que tal evento ocorra quando as condições hídricas de um período em questão estão abaixo das climaticamente esperadas.

Após o cálculo do balanço hídrico seqüencial decendial, foi calculada a média decendial do parâmetro ETR, representando uma condição climatologicamente esperada para um dado decêndio. A subtração entre o valor da ETR estimada em um dado período e sua média decendial dá origem ao parâmetro AH_ETR, que representa o desvio que uma dada condição estimada apresenta em relação a seu valor esperado. Foram ainda comparados valores do IPER com valores estimados da deficiência hídrica para as localidades em questão.

\section{RESULTADOS E DISCUSSÃO}

Na figura 1, verifica-se a a variação do parâmetro deficiência hídrica e a variação do IPER nas localidades de Campinas, Mococa, Pindorama e Ubatuba.

Os dados da figura 1 indicam uma concordância entre a variação da deficiência hídrica e do IPER em todas as localidades. Em Ubatuba, os decêndios de $21 / 1 / 04$ a 31/1/04 e de $1.0 / 1 / 05$ a $10 /$ 1/05, indicam valores de IPER de 0,8 e 1,2, respectivamente, e de deficiência hídrica de aproximadamente $1,5 \mathrm{~mm}$ em ambos os períodos.

Nota-se, portanto, que apesar de um ligeiro déficit no solo, em um período climatologicamente úmido, o índice não acusou condições de seca agrícola. Tal aparente incoerência pode ser mais bem entendida analisando-se a tabela 1.

Apesar da ocorrência de deficiência hídrica, foram estimados em ambos os casos, valores de ETR acima do patamar climatologicamente esperado. Entretanto, os altos valores de evapotranspiração potencial registrados nos períodos em questão, resultaram, mesmo com a ocorrência de precipitação pluvial, em uma ligeira deficiência hídrica no solo.

É evidente que se esse déficit entre a "saída" (ETP) e a "entrada" (precipitação) de água no sistema persistisse por um tempo maior, o armazemento de água no solo seria significativamente afetado, reduzindo as taxas de evapotranspiração real, a qual seria detectada pelo IPER. 

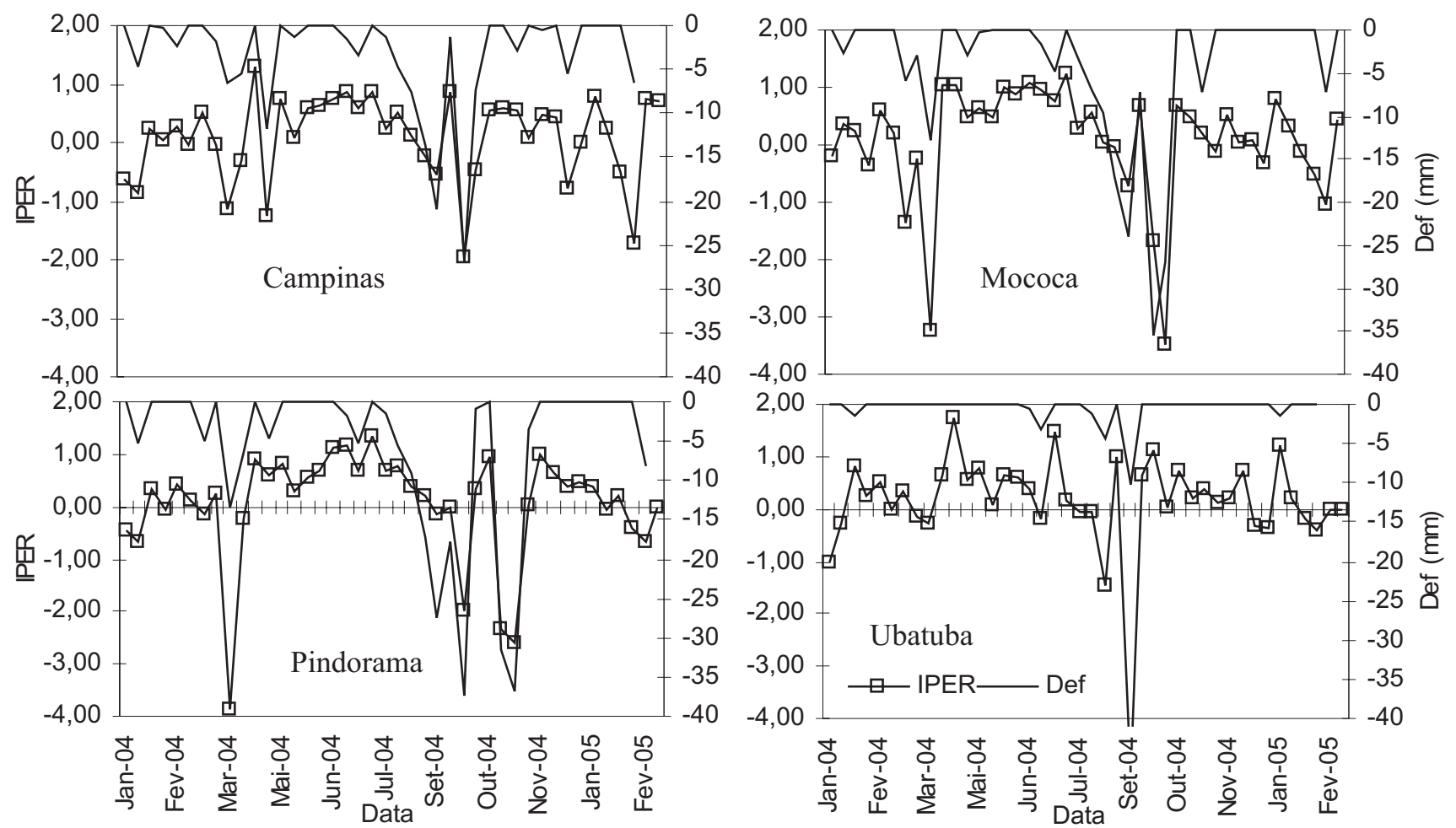

Figura 1. Variação do IPER em relação à variação da deficiência hídrica, estimada, segundo ThORNTWAITE e MATHER (1995) para Campinas, Mococa, Pindorama e Ubatuba entre janeiro de 2004 a fevereiro de 2005.

Tabela 1. Valores de evapotranspiração potencial (ETP), evapotranspiração real (ETR), índice padronizado de evapotranspiração real (IPER), evapotranspiração real climatologicamente esperada (ETRMed), anomalia da evapotranspiração real (AH_ETR), desvio-padrão das médias das ETR decendiais e da deficiência hídrica estimada, segundo THORNTWAITE e MATHER (1995)

\begin{tabular}{lcccccrr}
\hline Decêndio & ETP & ETR & IPER & ETRMed & AH_ETR & DP & Def. \\
\cline { 2 - 7 } & & & & & & & \\
$21-31 / 01 / 2004$ & 56,0 & 54,6 & 0,8 & 51,5 & 3,1 & 5,8 & $-1,5$ \\
$01-10 / 01 / 2005$ & 52,0 & 50,5 & 1,2 & 45,4 & 5,1 & 4,5 & $-1,5$ \\
\hline
\end{tabular}

A tabela 2 contém, para as regiões de Campinas, Jaú, Mococa, Pindorama, Ribeirão Preto e Ubatuba, valores de IPER, média de cada série decendial da ETR (ETRMed), AH_ETR, do desvio-padrão (DP) das séries de ETR, da ETR estimada em cada decêndio e da razão entre AH_ETR e o DP (AH_ETR/DP), no período de janeiro e fevereiro de 2005.

A coluna AH_ETR/DP representa o número de desvios-padrão que um dado valor de ETR, estimado em um dado decêndio, está cima ou abaixo do valor climatologicamente esperado (ETRMed) para o período em questão. Comparando-se a coluna IPER com AH_ETR/DP nota-se uma grande concordância entre os valores nelas existentes (Figura 2).

Por meio da figura 2, nota-se que o coeficiente angular $(0,9776)$ da equação resultante da regressão linear entre os dados da coluna IPER e da coluna AH_ETR/ DP, demonstradas na tabela 2, e o intercepto dessa mesma expressão $(0,0639)$ revelam que a variação dos parâmetros em questão está próxima à variação 1:1. Assim, pode-se concluir que o IPER é um índice de seca agrícola padronizado no tempo e no espaço, já que seus resultados estão relacionados a variação climatologicamente esperada da ETR em um local e período. 
Tabela 2. Valores do Índice Padronizado de Evapotranspiração Real (IPER) e evapotranspiração real (ETR), estimada segundo Thorntwaite e MATHer (1995), da ETR média e do desvio-padrão (DP) no período de janeiro a fevereiro de 2005, em Campinas, Jaú, Mococa, Pindorama, Ribeirão Preto e Ubatuba

\begin{tabular}{|c|c|c|c|c|c|c|c|c|c|c|c|c|}
\hline \multicolumn{6}{|c|}{ Campinas } & \multicolumn{7}{|c|}{ Jaú } \\
\hline Data & IPER & ETRMed & AH_ETR & DP & ETR & $\frac{\text { AH_ETR }}{\text { DP }}$ & IPER & ETRMed & AH_ETR & $\mathrm{DP}$ & ETR & $\frac{\text { AH_ETR }}{\text { DP }}$ \\
\hline $1 .^{\circ} / 01$ & 0,78 & 40,63 & 3,37 & 4,20 & 44,0 & 0,80 & 0,50 & 42,40 & 1,80 & 3,63 & 44,2 & 0,50 \\
\hline 11/01 & 0,22 & 42,72 & 1,28 & 4,35 & 44,0 & 0,29 & $-0,26$ & 44,15 & $-0,95$ & 3,44 & 43,2 & $-0,28$ \\
\hline $21 / 01$ & $-0,52$ & 46,84 & $-2,84$ & 4,50 & 44,0 & $-0,63$ & $-1,10$ & 47,63 & $-4,93$ & 3,99 & 42,7 & $-1,24$ \\
\hline $1 .^{\circ} / 02$ & $-1,73$ & 40,33 & $-8,33$ & 4,85 & 32,0 & $-1,72$ & 0,09 & 41,35 & 0,45 & 4,98 & 41,8 & 0,09 \\
\hline $11 / 02$ & 0,73 & 38,74 & 2,26 & 5,05 & 41,0 & 0,45 & 1,44 & 40,94 & 3,76 & 3,40 & 44,7 & 1,10 \\
\hline $21 / 02$ & 0,72 & 31,64 & 4,36 & 5,46 & 36,0 & 0,80 & 1,22 & 33,90 & 4,80 & 3,67 & 38,7 & 1,31 \\
\hline \multicolumn{7}{|c|}{ Mococa } & \multicolumn{5}{|c|}{ Pindorama } & \\
\hline $1 .^{\circ} / 01$ & 0,79 & 41,48 & 2,12 & 2,96 & 43,60 & 0,72 & 0,40 & 42,65 & 1,05 & 3,20 & 43,70 & 0,33 \\
\hline $11 / 01$ & 0,32 & 42,51 & 1,29 & 4,12 & 43,80 & 0,31 & $-0,04$ & 43,90 & 0,00 & 4,11 & 43,90 & 0,00 \\
\hline $21 / 01$ & $-0,12$ & 46,59 & $-1,49$ & 4,22 & 45,10 & $-0,35$ & 0,19 & 47,83 & $-0,63$ & 4,81 & 47,20 & $-0,13$ \\
\hline $1 .^{\circ} / 02$ & $-0,51$ & 39,87 & $-1,87$ & 3,89 & 38,00 & $-0,48$ & $-0,41$ & 40,83 & $-1,83$ & 5,11 & 39,00 & $-0,36$ \\
\hline $11 / 02$ & $-1,02$ & 38,97 & $-5,47$ & 4,91 & 33,50 & $-1,12$ & $-0,67$ & 41,46 & $-4,36$ & 4,34 & 37,10 & $-1,00$ \\
\hline $21 / 02$ & 0,46 & 33,13 & 2,57 & 4,93 & 35,70 & 0,52 & 0,27 & 34,13 & 1,67 & 5,61 & 35,80 & 0,30 \\
\hline \multicolumn{7}{|c|}{ Ribeirão Preto } & \multicolumn{5}{|c|}{ Ubatuba } & \\
\hline $1 .^{\circ} / 01$ & 0,66 & 41,27 & 2,13 & 3,14 & 43,40 & 0,68 & 1,20 & 45,38 & 5,12 & 4,55 & 50,50 & 1,13 \\
\hline $11 / 01$ & 0,76 & 42,44 & 3,96 & 4,68 & 46,40 & 0,85 & 0,20 & 45,89 & 2,01 & 9,12 & 47,90 & 0,22 \\
\hline 21/01 & 0,43 & 46,03 & 0,87 & 3,04 & 46,90 & 0,29 & $-0,19$ & 51,51 & $-2,71$ & 5,79 & 48,80 & $-0,47$ \\
\hline $1 .^{\circ} / 02$ & $-0,67$ & 40,58 & $-3,38$ & 5,10 & 37,20 & $-0,66$ & $-0,40$ & 43,69 & $-5,09$ & 9,84 & 38,60 & $-0,52$ \\
\hline $11 / 02$ & $-2,93$ & 39,63 & $-10,53$ & 3,49 & 29,10 & $-3,02$ & 0,05 & 42,61 & $-1,21$ & 8,33 & 41,40 & $-0,14$ \\
\hline $21 / 02$ & $-0,05$ & 32,86 & 0,14 & 4,26 & 33,00 & 0,03 & 0,02 & 35,23 & $-0,53$ & 7,87 & 34,70 & $-0,07$ \\
\hline
\end{tabular}

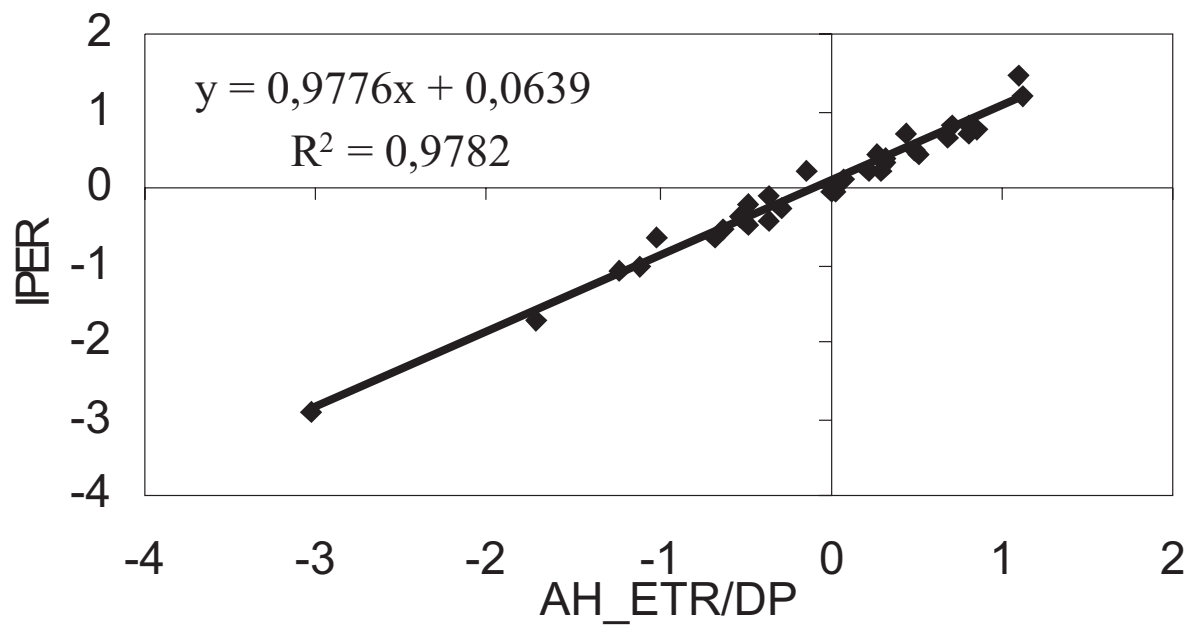

Figura 2. Regressão linear simples entre a variação do IPER e a variação da razão entre a anomalia de Evapotranspiração real e seu desvio-padrão (AH_ETR/DP) nos decêndios compreendidos entre $1 .^{\circ}$ de janeiro a 28 de fevereiro de 2005 , em Campinas, Jaú, Mococa, Pindorama, Ribeirão Preto e Ubatuba. 
Uma conseqüência da característica acima citada é a facilidade de interpretação dos resultados desse novo índice, visto que o valor do índice igual a -1 indica estar a ETR decendial, aproximadamente, a um desvio-padrão abaixo do valor esperado; o valor do índice igual a -2 indica estar a ETR decendial, aproximadamente, a 2 desvios-padrão abaixo do valor esperado e assim, sucessivamente, para qualquer região em qualquer decêndio.

Tal fato torna o IPER uma importante ferramenta no uso de políticas estaduais de combate e mitigação da seca agrícola no Estado de São Paulo.

Na tabela 3, verifica-se a variação do IPER em relação a uma variação hipotética de valores de ETR, quando são descontados 0, 1, 2, 3 e 4 desvios-padrão da ETR média no segundo decêndio de fevereiro e no primeiro decêndio de agosto nas localidades de Ribeirão Preto e Campinas, respectivamente em anos hipotéticos.

Tabela 3. Variação do índice padronizado de evapotranspiração real (IPER) em relação a uma variação hipotética de valores da evapotranspiração real (ETR), estimada segundo THORNTWAITE e MATHER (1995), quando são descontados 0, 1, 2, 3 e 4 desvios-padrão (DP) da ETR média no segundo decêndio de fevereiro, em Ribeirão Preto e no primeiro decêndio de agosto, em Campinas

\begin{tabular}{lcc}
\hline \multicolumn{3}{l}{ Ribeirão Preto, 11 a 20 de fevereiro } \\
\hline DP subtraídos & ETR $(\mathrm{mm})$ & IPER \\
0 & 32,86 & $-0,1$ \\
1 & 28,6 & $-1,1$ \\
2 & 24,4 & $-2,2$ \\
3 & 20,1 & $-3,3$ \\
4 & 15,9 & $-4,6$ \\
\hline Campinas, $1 .^{\circ}$ a 10 de agosto & & \\
\hline DP subtraídos & ETR (mm) & IPER \\
0 & 13,52 & 0,1 \\
1 & 8,6 & $-1,0$ \\
2 & 3,7 & $-2,4$ \\
\hline
\end{tabular}

Pela tabela 3, demonstra-se que em uma estação climatologicamente úmida, isto é, em períodos com valores médios mais altos de ETR, são potencialmente mais sujeitos a valores mais severos do IPER, do que as estações climatologicamente secas.

Na região de Ribeirão Preto, por exemplo, no segundo decêndio de fevereiro (estação úmida), um valor de 24,4 mm de ETR gera um IPER de -2,2, enquanto na região de Campinas em (estação seca) um valor de $-2,4$ do índice é obtido apenas após o valor da ETR atingir 3,7 mm.
De maneira geral, no Estado de São Paulo, o período de maior demanda hídrica das culturas coincide com a estação mais chuvosa do ano. Dessa forma, a diminuição abrupta no regime de precipitação pluvial no Estado e na estação em questão (veranico), tem um potencial de dano maior às culturas do que se tal diminuição ou escassez de chuva ocorrer em um período climatologicamente seco, já que esse último coincide, normalmente, com estádios fenológicos de relativa baixa demanda hídrica das culturas. Assim, torna-se desejável que o índice em questão, por ser voltado aos interesses agrícolas, seja mais sensível em períodos climatologicamente úmidos. Tal característica decorre do fato de uma seca agronômica estar relacionada a fases críticas do desenvolvimento/ crescimento dos vegetais.

Na figura 3, observa-se a variação do IPER em relação à variação de valores de AH_ETR nas localidades de Campinas, Jaú, Mococa, Pindorama, Ribeirão Preto e Ubatuba.

Analisando-se a figura 3, nota-se uma concordância bastante significativa entre a variação do índice Padronizado de Evapotranspiração Real e a variação de AH_ETR nas quatro localidades. Tal concordância é mais um indicativo de ser o IPER uma ferramenta consistente para a quantificação/ padronização das condições de seca agrícola no Estado de São Paulo.

Devido ao fato de ser ajustado à distribuição normal, o Índice Padronizado de Evapotranspiração Real representa uma freqüência de ocorrência de suas classes de valores semelhante nas diversas localidades em que é aplicado. Valores iguais ou superiores a zero indicam uma freqüência próxima a $60 \%$; valores entre 0 e $-1,25 \%$; valores entre -1 e $-2,10 \%$; valores entre -2 e $-3,4 \%$; valores abaixo de $-3,2 \%$.

O IPER é uma ferramenta que quantifica o desvio de um dado valor estimado de ETR tem em relação ao seu valor climatologicamente esperado. Não é, portanto, voltado ao estudo de "tipos de seca" que consideram o excedente hídrico gerado em uma região como elemento importante em seu estudo como, por exemplo, a seca hidrológica. Em outras palavras, de acordo com método de ThornthWAite e Mather (1955), quando o valor de ETR, de um determinado período, é igual ao da ETP e o solo está em seu armazenamento máximo, o excedente hídrico passa a ser gerado. Entretanto, esse último parâmetro não permanece disponível às culturas, não sendo, portanto, considerado em um estudo de seca agrícola. 

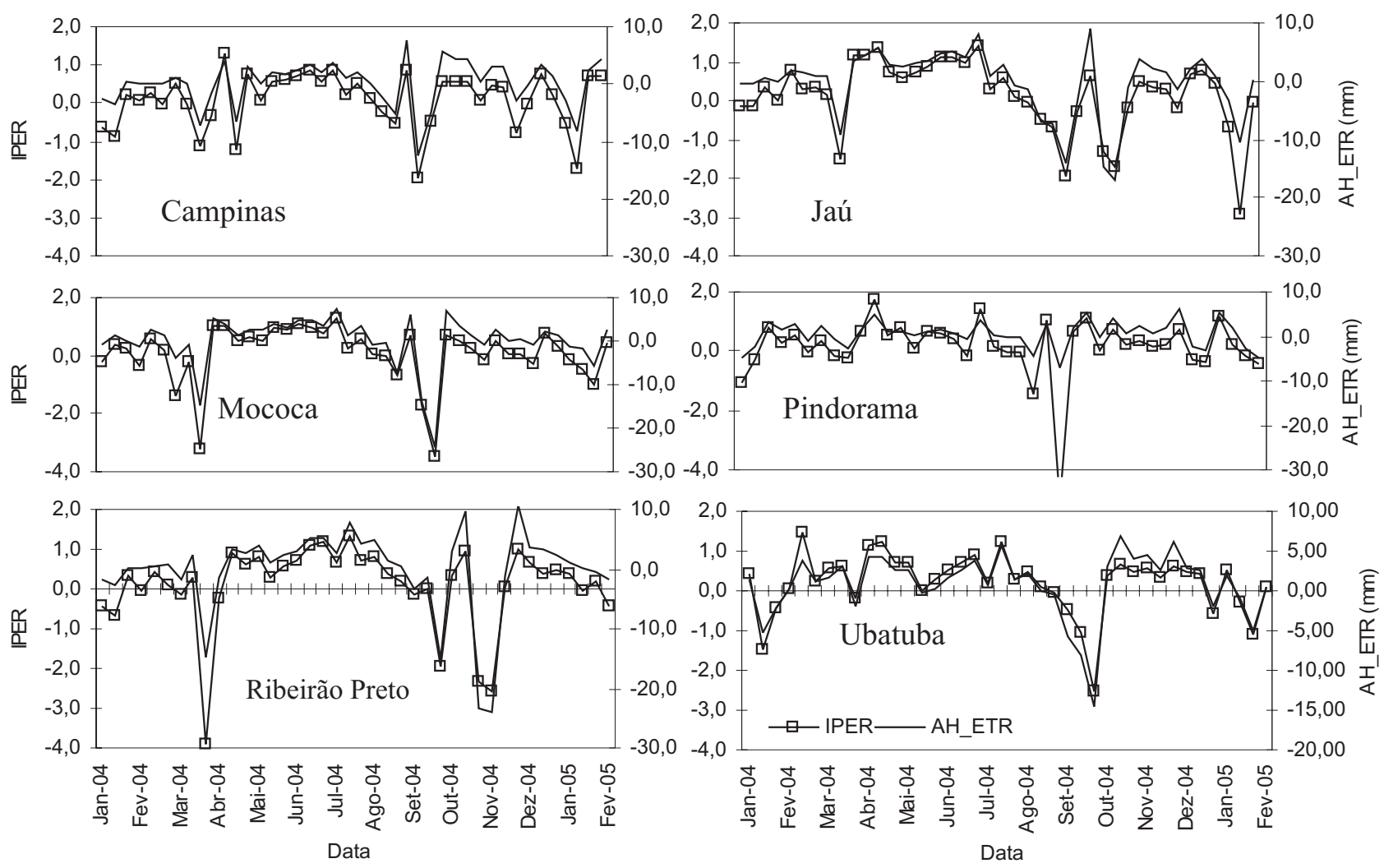

Figura 3. Variação do IPER em relação à variação de valores de ETR nas localidades de Campinas, Jaú, Mococa,

Pindorama, Ribeirão Preto e Ubatuba.

\section{CONCLUSÕES}

1. As análises comprovam que o Índice Padronizado de Evapotranspiração Real (IPER) é uma ferramenta consistente para o monitoramento/ padronização "próximo ao tempo real" da seca agrícola no Estado de São Paulo.

2. Devido ainda à facilidade de interpretação de seus resultados, o IPER deve ser utilizado, em programas estatais, como ferramenta auxiliar nas tomadas de decisão de medidas de combate aos efeitos da seca agrícola.

\section{REFERÊNCIAS}

ABRAMOWITZ, M., STEGUN, I.A. Handbook of mathematical function. Dover, 1965. p.1046.

BLAIN, G. C. Avaliação e adaptação do Índice de Severidade de Seca de Palmer (PDSI) e do Índice Padronizado de Precipitação (SPI) às condições climáticas do Estado de São Paulo. 2005. 120 f. Dissertação (Mestrado em Agricultura Tropical e Sub-tropical) - Instituto Agronômico, Campinas, SP.
BRUNINI, O., BLAIN, G. C., BRUNINI, A. P., SANTOS, R. L.,BRIGANTE, R. S., ALMEIDA, E. L: Avaliação do índice de severidade de Seca de Palmer para a quantificação da seca agrícola no Estado de São Paulo. In: CONGRESSO BRASILEIRODE METEOROLOGIA, 12., 2002, Foz do Iguaçu. Anais... Foz do Iguaçu: Sociedade Brasileira de Meteorologia, 2002. p. 1140-1147. (CD-ROM)

HAYES, M. J.;SVOBODA, M.D.; WILHITE, D.A.;VANYARKHO, O.V. Monitoring the 1996 drought using the Standardized Precipitation Index. Bulletin of the American Meteorology Society, Boston, v. 80, n. 3, p.429-438, 1999.

HEIM JUNIOR, R.R. A review of twentieth: century drought indices used in the United States. Bulletin of the American Meteorology Society, Boston, v. 83, n.8, p.1149-1163, 2002.

IPCC, Climate Change 2001. Impacts, Adaptation and Vulnerability. Contribution of Working Group 2 to the Third Assessment Report of the Intergovernmental Panel on Climate Change. In: HOUGHTON, J.T. (Ed.). Cambridge: Cambridge University Press, 2001.

KEYANTASH, J. DRACUP, J.A. The quantification of drought: An evaluation of drought indices. Bulletin of the American Meteorology Society, Boston, v. 83 n.8, p.1167-1180, 2004. 
MCKEE, T.B.; DOESKEN, N.J.; KLEIST, J. The relationship of drought frequency and duration to times scale. In: CONFERENCE ON APPIED CLIMATOLOGY, 8., 1993, Boston. Anais... Boston: American Meteorological Society, 1993. p.179- 184. (Preprints)

PALMER, W.C. Keeping track of crop moisture conditions, nationwide: the new Crop Moisture Index. Weatherwise, v. 21, p.156-161, 1968.

RASMUSSEN, E.M.;DICKINSON, R.E.; KUTZBACH, J.E.; CLEAVELAND,M.K. Climatology. In: MAIDMEMT, D.R. Handbook of Hydrology. New York: McGraw-Hill, 1993. cap. 2, p.1-44

THORNTHWAITE, C.W.; MATHER, J.R. The water balance. Centerton: Drexel Institute of Technology - Laboratory of Climatology, 1955. 104 p. (Publications in Climatology, v.8, n.1)
WILHITE, D. A. Drought as a natural hazard: conceptions and definitions. In: WILHITE, D. A. Drought: A global assessment. London: Routledge, 2000. p.111-120.

WILHITE, D. A. Government response to drought in the United States: with particular reference to the Great Plains. Jounal of Climate and Applied Meteorology, v. 22, p. 40-50, 1982.

WILHITE, D. A.; GLANTZ, M.H. Understanding the drought phenomenon: The role definations. In: WILHITE et al. Planning for drought toward a reduction of societal vulnerability. Colorado: Westview, 1987. cap. 2, p. 11-14.

WORLD METEOROLOGICALORGANIZATION. Drought and agriculture. Geneva, Switzerland: WMO, 1975. 127 p. (Tech. Note 138, Publ. WMO-392) 